\title{
Impact of adverse drug reaction and predictivity of quality of life status in tuberculosis
}

\section{To the Editors:}

In Canada, people diagnosed with active tuberculosis (TB) disease are routinely treated with isoniazid, rifampin, pyrazinamide and ethambutol. Although effective, the treatment is associated with significant adverse drug reactions (ADRs). Current knowledge of these ADRs has focused on their frequency and clinical natures [1, 2]. However, reporting clinical natures of ADRs is not adequate to fully reflect their impact on patients' health status. In recent years, patientreported outcomes, such as health-related quality of life (HRQoL), are increasingly appreciated in evaluating the impact of illnesses and the effectiveness of medical interventions [3]. Therefore, we intended to investigate the impact of anti-TB treatment-induced ADRs on patients' HRQoL and to examine the association between baseline HRQoL status and the likelihood of ADRs during the subsequent treatment.

In British Columbia (Canada), TB patients are seen monthly through TB control clinics managed by the British Columbia Center for Disease Control (BCCDC). Monthly laboratory tests are done to monitor patients' liver and renal function and haematological status. Tolerance to medications is evaluated during clinic visits. Medical records are kept in the integrated Public Health Information System (iPHIS).

This study partially represents a longitudinal HRQoL study, where English-speaking adults with newly initiated treatment for active TB disease or latent TB infection were recruited from BCCDC during 2005 and 2006. Ethics approval was obtained from the Behavioural Research Ethics Board of the University of British Columbia (Vancouver, BC, Canada). Participants provided informed consent. For the present study, active TB patients were considered eligible if they further met the criteria: 1) had no pre-existing liver problems or no other known severe health conditions before the treatment; 2) completed $\geqslant 3$ months of treatment; and 3) completed both baseline and 3-month HRQoL assessments.

HRQoL was measured at baseline, 3 months and 6 months of the treatment using the Short-Form 36 version 2 (SF-36 v2) [4], as there is no well-validated TB-specific questionnaire in the literature. SF-36 measures eight dimensions: physical functioning $(\mathrm{PF})$, role-physical (RP), bodily pain (BP), general health $(\mathrm{GH})$, vitality (VT), social functioning (SF), role-emotional (RE) and mental health $(\mathrm{MH})$. From these subscales, a physical component summary (PCS) and a mental component summary (MCS) can be determined [4]. As the majority of ADRs occurred within the first 3 months of anti-TB treatment $[1,2]$, only baseline and 3-month SF-36 outcomes were used for analyses.

From the iPHIS database, physician narratives and nurse notes were retrospectively reviewed to identify anti-TB medicationrelated ADRs. The criteria we have used previously to assess the possibility and severity of ADRs were applied here [2]. All definite, possible and probable ADRs during the first 3 months of the treatment were included and classified by severity: an ADR was defined as major if it led to discontinuation of routine treatment and/or required additional treatment for the symptoms; an ADR was minor if no additional medical interventions were taken. Patients were then categorised into the following three groups. 1) No ADR: never had any ADR. 2) Minor ADR: only developed minor ADRs. 3) Major ADR: developed at least one major ADR.

ANOVA, Pearson's Chi-squared test or Fisher's exact test was used to compare the baseline sociodemographic differences between the three groups. SF-36 scores were calculated and transformed using norm-based scoring that resulted in a mean of 50 and standard deviation of 10 in the general population [4]. A higher SF-36 score indicates a better HRQoL outcome. Multiple linear regression models were constructed to explore the impact of ADRs on the 3-month HRQoL. The dependent variable was each one of the SF-36 scales (eight subscales, PCS and MCS). In each model, two independent variables were included to represent the presence/absence of major ADRs and minor ADRs respectively. Baseline SF-36 score, age, sex, race, marital status, smoking and the presence of comorbidities were adjusted for in each model. Ordinal logistic regression was applied to examine the association between baseline SF-36 scores and the occurrence of ADRs during the first 3 months. The dependent variable indicating the occurrence of ADRs had three levels: no ADR, minor ADR and major ADR. Then we added each of the baseline SF-36 scores, as a predictor variable, to separate models. Age, sex, race, marital status, smoking and the presence of comorbidities were included in each model. Considering their clinical importance, all covariates were kept in final fitted regression models, regardless of their statistical significance. All analyses were performed using software SAS (version 9.0; SAS Institute Inc, Cary, NC, USA).

Among the 104 active TB patients recruited in the HRQoL study, 89 further met the present study's inclusion criteria and were included. Their average age was $49.2 \mathrm{yrs}$, with $56 \%$ being female. $71 \%$ were ethnically Asian. Of the 89 subjects, $41(46 \%)$ reported having other health conditions, such as high blood pressure, diabetes, back problems, arthritis and asthma. During the first 3 months of treatment, 21 patients $(24 \%)$ developed at least one major ADR and another 29 (33\%) experienced only minor ADRs. Common ADRs included skin rash and/or pruritus (35\%), gastrointestinal symptoms $(30 \%)$, liver damage (including mild liver enzyme elevation and hepatitis) (23\%), paresthesia $(18 \%)$, fatigue/weakness $(18 \%)$, visual disturbance $(17 \%)$ and joint/muscle pain $(17 \%)$. The most common ADR leading to treatment discontinuation was liver enzyme elevation (11\%). Sociodemographic differences were observed across the three groups (no ADR, minor ADR and major ADR), e.g. females 
were more likely to develop ADRs than males. However, none of these differences were statistically significant.

Baseline and 3-month SF-36 scores are summarised in table 1. At 3 months, all SF-36 scores were monotonically decreasing across the three groups. After adjusting for sociodemographic factors and baseline SF-36 scores, compared to those who had no ADRs, subjects who developed major ADRs had significantly lower 3-month scores on PF $(p=0.03)$, VT $(p=0.01), \mathrm{MH}$ $(p=0.01)$ and MCS $(p=0.03)$. No significant differences were found between subjects who experienced only minor ADRs and those who had no ADRs. As seen in table 1, subjects who developed major ADRs during the 3 months of treatment scored lowest at baseline, whereas those who had no ADRs scored highest. Ordinal logistic regression analyses showed that subjects who scored lower on SF-36 at baseline were significantly associated with a higher risk of developing ADRs during the treatment, after adjusting for sociodemographic factors. For example, for a one-unit increase in baseline MCS score, the odds of developing ADRs (minor or major) would be reduced by $6 \%(\mathrm{OR} 0.94, \mathrm{p}=0.001)$, and the odds of having major ADRs versus having no ADRs and minor ADRs would be reduced by $6 \%$ as well (the proportional odds assumption).

This study is the first to examine the anti-TB treatmentinduced ADRs in terms of HRQoL experienced by patients. We found that anti-TB treatment ADRs had substantial impacts on patients' HRQoL, and that poor baseline HRQoL status was associated with a higher possibility of developing ADRs during the subsequent treatment.

Our results showed that developing major ADRs led to significant reduction on two mental health subscales (VT and $\mathrm{MH})$, the mental health summary (MCS) and one physical subscale (PF) of SF-36. Experiencing minor ADRs showed some negative impacts, but none were statistically significant. This suggests that severe ADRs result in more decrements in HRQoL; experiencing ADRs seems to be more of a mental well-being burden than a physical one. We also observed that patients with lower baseline SF-36 scores had a higher risk of developing ADRs during the first 3 months of treatment, after adjusting for important sociodemographic factors. HRQoL measures capture patients' self-perceived health status, functioning and well-being. Poor baseline HRQoL status may reflect known or unknown physical and mental health problems and individual psychological coping mechanisms, which would predispose to a higher risk of unfavourable outcomes. Our results suggest that baseline HRQoL measurements could potentially help identify those patients who would eventually develop an ADR to the anti-TB treatment. This finding could help plan quality healthcare management and justify resource allocation, e.g. improving the baseline HRQoL of those high-risk patients through lifestyle modification consultation and social-psychological support would promote the outcome to medical treatment. Future prospective studies are needed to better understand the predictive value of HRQoL status and to determine whether it could be manipulated for therapeutic purpose [5-7].

There are some limitations to our study. First, ADRs were retrospectively collected by reviewing administrative medical records, which usually are not established for the purpose of

\begin{tabular}{|c|c|c|c|c|}
\hline SF-36 v2 scales & Subjects $n$ & Baseline & 3 months & p-value \\
\hline \multicolumn{5}{|c|}{ Physical functioning } \\
\hline No ADR & 38 & $45.2 \pm 14.4$ & $46.9 \pm 13.5$ & \\
\hline Minor ADR & 29 & $42.6 \pm 12.3$ & $44.7 \pm 11.9$ & 0.75 \\
\hline Major ADR & 20 & $33.6 \pm 13.2$ & $33.8 \pm 11.9^{*}$ & 0.03 \\
\hline \multicolumn{5}{|l|}{ Role-physical } \\
\hline No ADR & 36 & $45.0 \pm 13.4$ & $47.1 \pm 13.0$ & \\
\hline Minor ADR & 29 & $36.2 \pm 15.1$ & $41.0 \pm 14.7$ & 0.70 \\
\hline Major ADR & 18 & $29.4 \pm 13.6$ & $36.7 \pm 11.1$ & 0.95 \\
\hline \multicolumn{5}{|l|}{ Bodily pain } \\
\hline No ADR & 39 & $53.9 \pm 11.4$ & $55.9 \pm 9.6$ & \\
\hline Minor ADR & 28 & $52.8 \pm 9.2$ & $53.2 \pm 8.8$ & 0.87 \\
\hline Major ADR & 20 & $44.1 \pm 15.6$ & $45.5 \pm 13.7$ & 0.26 \\
\hline \multicolumn{5}{|l|}{ General health } \\
\hline No ADR & 39 & $46.2 \pm 9.5$ & $47.7 \pm 9.0$ & \\
\hline Minor ADR & 28 & $44.2 \pm 8.5$ & $47.1 \pm 8.7$ & 0.44 \\
\hline Major ADR & 20 & $41.2 \pm 8.0$ & $41.1 \pm 8.2$ & 0.07 \\
\hline \multicolumn{5}{|l|}{ Vitality } \\
\hline No ADR & 39 & $46.9 \pm 12.0$ & $50.1 \pm 9.5$ & \\
\hline Minor ADR & 28 & $42.4 \pm 12.0$ & $46.2 \pm 8.6$ & 0.41 \\
\hline Major ADR & 20 & $38.5 \pm 10.6$ & $39.1 \pm 10.8^{\star}$ & 0.01 \\
\hline \multicolumn{5}{|l|}{ Social functioning } \\
\hline No ADR & 39 & $44.8 \pm 11.6$ & $46.5 \pm 11.1$ & \\
\hline Minor ADR & 28 & $35.6 \pm 14.8$ & $43.4 \pm 11.4$ & 0.60 \\
\hline Major ADR & 20 & $32.6 \pm 13.6$ & $36.4 \pm 13.1$ & 0.12 \\
\hline \multicolumn{5}{|l|}{ Role-emotion } \\
\hline No ADR & 37 & $40.6 \pm 16.4$ & $43.9 \pm 16.4$ & \\
\hline Minor ADR & 29 & $32.1 \pm 18.9$ & $38.8 \pm 15.8$ & 0.66 \\
\hline Major ADR & 18 & $22.4 \pm 15.8$ & $31.7 \pm 13.7$ & 0.68 \\
\hline \multicolumn{5}{|l|}{ Mental health } \\
\hline No ADR & 38 & $47.8 \pm 9.0$ & $50.3 \pm 8.5$ & \\
\hline Minor ADR & 26 & $41.0 \pm 12.7$ & $46.2 \pm 7.6$ & 0.83 \\
\hline Major ADR & 20 & $39.2 \pm 9.5$ & $39.2 \pm 13.2^{*}$ & 0.01 \\
\hline \multicolumn{5}{|l|}{ Physical summary } \\
\hline No ADR & 35 & $49.2 \pm 11.6$ & $50.6 \pm 10.5$ & \\
\hline Minor ADR & 26 & $47.0 \pm 9.1$ & $48.6 \pm 9.7$ & 0.32 \\
\hline Major ADR & 18 & $38.4 \pm 10.9$ & $40.3 \pm 10.2$ & 0.52 \\
\hline \multicolumn{5}{|l|}{ Mental summary } \\
\hline No ADR & 35 & $44.7 \pm 9.5$ & $47.7(9.1)$ & \\
\hline Minor ADR & 26 & $36.2 \pm 14.8$ & $43.0 \pm 8.7$ & 0.96 \\
\hline Major ADR & 18 & $32.5 \pm 11.6$ & $36.3 \pm 12.5^{\star}$ & 0.03 \\
\hline
\end{tabular}

Data are presented as mean $\pm \mathrm{SD}$, unless otherwise stated. v2: version 2. ${ }^{\#}: \mathrm{p}$ values are based on the multiple regression analyses, adjusting for baseline SF36 score, age, sex, race, marital status, smoking and comorbidity. Subjects who had no ADRs are the reference. ${ }^{*}: p<0.05$.

research and may not be comprehensive. Second, the TB population may be exposed to various risk factors. Although we controlled for some important sociodemographic factors in our analyses, there may still be unmeasured confounders that we did not capture. Finally, our inadequate sample size might prevent us from exploring more significant findings. Despite these limitations, as the first study on this topic, we believe the current report is a good starting point for future research. 


\section{N. Guo*, F. Marra*,\#, J.M. FitzGerald", R.K. Elwood ${ }^{\#, \oplus}$ and C.A. Marra*}

*Faculty of Pharmaceutical Sciences, University of British Columbia, "British Columbia Centre for Disease Control, and "Faculty of Medicine, University of British Columbia, Vancouver, BC, Canada.

Correspondence: C.A. Marra, Faculty of Pharmaceutical Sciences, University of British Columbia, 2146 East Mall, V6T $1 Z 3$ Vancouver, BC, Canada. E-mail: carlo.marra@ubc.ca

Statement of Interest: None declared.

\section{REFERENCES}

1 Forget EJ, Menzies D. Adverse reactions to first-line antituberculosis drugs. Expert Opin Drug Saf 2006; 5: 231-249.
2 Marra F, Marra CA, Bruchet $\mathrm{N}$, et al. Adverse drug reactions associated with first-line anti-tuberculosis drug regimens. Int $J$ Tuberc Lung Dis 2007; 11: 868-875.

3 Guyatt GH, Feeny DH, Patrich DL. Measuring health-related quality of life. Ann Intern Med 1993; 118: 622-629.

4 Ware JE Jr, Kosinski M, Bjorner JB, et al. User's manual for the SF36v2 ${ }^{\circledR}$ Health Survey. $2^{\text {nd }}$ edn. Lincoln, RI: Quality Metric Incorporated, 2007.

5 Gold M, Franks P, Erickson P. Assessing the health of the nation. The predictive validity of a preference-based measure and self-rated health. Med Care 1996; 34: 163-177.

6 Franks P, Gold MR, Fiscella K. Sociodemographics, self-rated health, and mortality in the US. Soc Sci Med 2003; 56: 2505-2514.

7 Sehlen S, Lenk M, Hollenhorst H, et al. Quality of Life (QoL) as Predictive Mediator Variable for Survival in Patients with Intracerebral Neoplasma During Radiotherapy. Onkologie 2003; 26: 38-43.

\section{Development of a standardised tool to survey MDR-/XDR-TB case management in Europe}

\section{To the Editors:}

The emergence of multidrug-resistant tuberculosis (MDR-TB, defined as in vitro resistance to isoniazid and rifampicin) [1-5] and extensively drug-resistant TB (XDR-TB, defined as in vitro drug resistance to isoniazid and rifampicin plus any fluoroquinolone and at least one of the injectable drugs: capreomycin, kanamycin or amikacin) represents a major threat to TB control at the global level [1-5].

In 2007, the World Health Organization (WHO) estimated a prevalence of 511,000 MDR-TB cases (with 150,000 deaths) and 50,000 XDR-TB cases (and 30,000 deaths) [1]; 14 out of 19 highMDR-TB burden territories are located in Former Soviet Union (FSU) countries [1].

$\mathrm{XDR}-\mathrm{TB}$ is a manmade product, resulting, in essence, from clinical mismanagement of newly diagnosed pan-susceptible TB cases as well as MDR-TB cases [1,2]. There is evidence that suboptimal TB case management in parts of Europe contributes to the development of resistance to the XDR-TB defining drugs [4-7].

In spite of the growing amount of public awareness about TB drug resistance, the essential variables necessary to fully understand MDR-TB and XDR-TB are unfortunately not systematically collected, analysed and reported in published studies [2, 8]. Although the international community is providing a rapid response to XDR-TB (formulation of an emergency plan [9]; development of an instrument to support implementation and scale-up of national strategies and to assess programmatic needs [10]), no standardised and comprehensive tool is available to survey the key factor responsible for the emergence of MDR-/ XDR-TB: inappropriate TB case management.
The aim of this letter is to describe the process undertaken by the Tuberculosis Network European Trialsgroup (TBNET) in collaboration with the European Centre for Disease Prevention and Control (ECDC) to develop a standardised tool to survey MDR-/XDR-TB case management in the European Union/ European Economic Area (EU/EEA) countries and the validation process used to finalise the tool.

This instrument was devised to survey original clinical records of susceptible TB and MDR-/XDR-TB cases to ascertain if case management activities were performed according to established guidelines and national regulations [11, 12]. It was not designed to detect pathogenetic mechanisms or risk factors involved in the emergence of drug-resistance in selected TB cases.

The tool identifies the most critical gaps in susceptible TB/ MDR-/XDR-TB case management that need to be addressed urgently to prevent adverse outcomes for both individual patient and public health.

Data elements were organised taking into account the following. 1) Structure and content of clinical records in a sample of European countries participating in the ECDC European survey on case management of MDR-TB (five EU countries, representing different $\mathrm{TB}$ and MDR-TB incidence: one high TB/high MDR-TB; two low TB/low MDR-TB in Northern and Southern Europe; one high TB/intermediate MDR-TB; and one intermediate TB/low MDR-TB). 2) The recommendations of the TBNET systematic review on XDR-TB management [2]: a) prospective study design, standardised, internationally accepted definitions, quality-controlled laboratory testing for all first- and second-line drugs defining XDRTB; agreed-upon set of standard variables allowing for comparison of approaches and results across studies; b) the 